\title{
Eficacia de biofertilizantes en la producción de variedades de frejol
}

\author{
Effectiveness of biofertilizers in the production of bean varieties \\ Eulalio Morel López*1, Ruth Esther Pistilli¹, Ever Barrios Valiente1, Oscar Caballero Casuriaga ${ }^{1}$, \\ Amílcar Servin ${ }^{1}$, Modesto Osmar Dasilva ${ }^{1}$, Wilfrido Lugo ${ }^{1}$, Álvaro Huerta ${ }^{1}$
}

\section{RESUMEN}

El objetivo del estudio fue evaluar la eficacia de la aplicación foliar de biofertilizantes en la producción de variedades de frejol. El experimento se realizó en el distrito de Belén, Concepción, Paraguay, en el ciclo productivo 2019. Se utilizó un diseño en bloques completos al azar (DBCA) dispuesto en parcelas subdivididas. La parcela principal correspondió a las variedades de frejol (negro y carioca) y la parcela secundaria a los biofertilizantes ( repeticiones. En todos los tratamientos, excepto el testigo, se aplicó el biofertilizante $(500 \mathrm{ml})$ disuelto en 20 litros de agua en tres oportunidades (20 días después de la emergencia al estado de plántula, antes de la floración y en formación de vainas). Las determinaciones estudiadas fueron altura de plantas a los 30, 50 y 70 DDE, número de vainas por planta, número de granos por vaina, peso de 1000 granos y rendimiento del cultivo. Los datos fueron sometidos al análisis de varianza (ANAVA) y las medias de las variables se compararon por el test de Tukey al 5\% de probabilidad de error. Los resultados arrojados por el experimento indican la eficacia de los biofertilizantes en la producción de variedades de frejol de forma significativa en todas las determinaciones evaluadas. Se observaron interacciones significativas de los factores en la altura a los $70 \mathrm{DDE}$, número de vainas y rendimiento.

Palabras clave: Phaseolus vulgaris L., aplicación foliar, frejol negro, frejol carioca.

\begin{abstract}
The objective of the study was to evaluate the effectiveness of the foliar application of biofertilizers in the production of broad bean varieties. The experiment was carried out in the district of Belén, Concepción, Paraguay, in the 2019 production cycle. A randomized complete block design (DBCA) arranged in subdivided plots was used, the main plot corresponding to the bean varieties (black and carioca), and the plot secondary to Biofertilizers (without biofertilizer, biofertilizer 1 and biofertilizers 2) with four replications. In all treatments, except the control, the biofertilizer $(500 \mathrm{ml})$ dissolved in 20 liters of water was applied three occasions (20 days after emergence to the seedling stage, before flowering and in pod formation). The determinations studied were; plant height at 30, 50 and 70 DDE, number of pods per plant, number of grains per pod, weight of 1000 grains and crop yield. The data were submitted to the analysis of variance (ANAVA) and the means of the variables were compared by the Tukey test with a 5\% probability of error. The results obtained by the experiment indicate the efficiency of Biofertilizers in the production of varieties of bean in a significant way in all the determinations evaluated. Significant interactions of the factors were observed in the height at 70 DDE, number of pods and yield.
\end{abstract}

Key words: Phaseolus vulgaris $L$., foliar application, black bean, carioca bean.

\section{Introducción}

El frejol (Phaseolus vulgaris L.) es una leguminosa de grano, perteneciente a la familia Fabaceae, cuya importancia económica y social se debe a su contenido en proteínas, fibras naturales y otros elementos en la dieta de la población, además de ser un buen complemento de los cereales y otras fuentes principales de carbohidratos (León et al., 2008).
Muchos factores influyen en los bajos rendimientos de los cultivos. Entre los aspectos que deben ser mejorados, el manejo de fertilización de la planta merece atención apropiada, ya que con el suministro adecuado y equilibrado de nutrientes para el frejol se puede contribuir a un aumento significativo en la productividad del cultivo (Andrade et al., 2004). La producción intensiva de alimentos exige el manejo adecuado de suelo para

$1 \quad$ Universidad Nacional de Concepción, Facultad de Ciencias Agrarias, Concepción, Paraguay.
* Autor por correspondencia: lopezeulalio@ hotmail.com 
garantizar la productividad y la sustentabilidad ambiental. Una de las alternativas es la utilización de residuos orgánicos para el desarrollo del cultivo, disminuyendo así la dependencia de fertilizante mineral (Galbiatti et al., 2011).

El biofertilizante es el proceso final de degradación de la materia orgánica y actúa nutricionalmente sobre el metabolismo vegetal. Los biofertilizantes tienen alta actividad microbiana y bioactiva y son capaces de proporcionar mayor protección y resistencia a la planta contra el ataque de agentes externos, además de una mayor actividad biodinámica en el suelo (Medeiros et al., 2003).

Las técnicas de biofertilizantes establecen importantes estrategias para el aprovechamiento eficiente y racional de los materiales agrícolas, sin necesidad de generar impactos económicos o climáticos adversos que puedan deteriorar los recursos hídricos y el medio ambiente. Por otro lado, los costos de producción y aplicación de biofertilizantes son menores que los de fertilizantes químicos (Carvajal y Mera, 2010).

Por tanto, ante la necesidad de disponer de tecnología alternativa e inducir a los pequeños productores a enfocarse en una producción saludable, se propuso evaluar el comportamiento de variedades del cultivo de frejol sometidas a la aplicación foliar de biofertilizantes a base de ingredientes naturales.

\section{Materiales y métodos}

El experimento se realizó en el distrito de Belén, departamento de Concepción, Paraguay., durante el periodo comprendido entre mayo y agosto de 2019. Sus coordenadas geográficas son: latitud S $23^{\circ} 27^{\prime} 58^{\prime \prime}$ y longitud O $57^{\circ} 15^{\prime} 43^{\prime \prime}$. Altitud media $79 \mathrm{msnm}$.

El clima de la región se caracteriza por presentar una temperatura promedio de $24{ }^{\circ} \mathrm{C}$ con máximas que pueden llegar hasta $\operatorname{los} 45^{\circ} \mathrm{C}$ en verano y mínima de hasta $6{ }^{\circ} \mathrm{C}$ en invierno, con leves incidencias de heladas. La precipitación media anual es de $1.300 \mathrm{~mm}$ (DMH, 2018). Los datos fueron obtenidos de la estación meteorológica localizada en la Facultad de Ciencias Agrarias, considerando la temperatura media y precipitación durante el experimento (Figura 1).

El suelo fue clasificado como orden Alfisol y subgrupo Mollic Paleudalf (López et al., 1995). Los atributos químicos del suelo, en la profundidad de 0-20 $\mathrm{cm}$ fueron: $\mathrm{P}\left(\mathrm{Mehlich}^{-1}\right)$ : $3,73 \mathrm{mg} \mathrm{dm}^{-3}$; M.O.: 11,16 $\mathrm{g} \mathrm{dm}^{-3} ; \mathrm{pH}\left(\mathrm{CaCl}_{2}\right)$ : 4,84; K: 0,05 $\mathrm{cmol} \mathrm{dm}^{-3}$; Ca: 2,77 $\mathrm{cmol} \mathrm{dm}^{-3}$; Mg: 0,34 $\mathrm{cmol} \mathrm{dm}^{-3} ; \mathrm{H}+\mathrm{Al}: 2,56 \mathrm{cmol} \mathrm{dm}^{-3} ; \mathrm{SB}$ : $3,19 \mathrm{cmol} \mathrm{dm}^{-3}$, CIC: $5,75 \mathrm{cmol} \mathrm{dm}^{-3} \mathrm{y}$ V: $55,47 \%$.

El diseño experimental utilizado fue en Bloques Completos al Azar (DBCA) con parcelas subdivididas dispuestos en esquema factorial ( $2 \times 3)$, con cuatro repeticiones. La parcela principal correspondió a la variedad de frejol (negro, carioca) y parcela secundaria a los biofertilizantes (sin biofertilizante, biofertilizante 1 y biofertilizante 2). La composición de los productos es la siguiente: Biofertilizante 1: Lactobacillus, levaduras y actinomicetos. Biofertilizante 2: Lactobacillus, levaduras, actinomicetos, pseudomonas, Azospirillum y Bradyrhizobium. Cada parcela tuvo una dimensión de $16 \mathrm{~m}^{2}$, con 4 metros
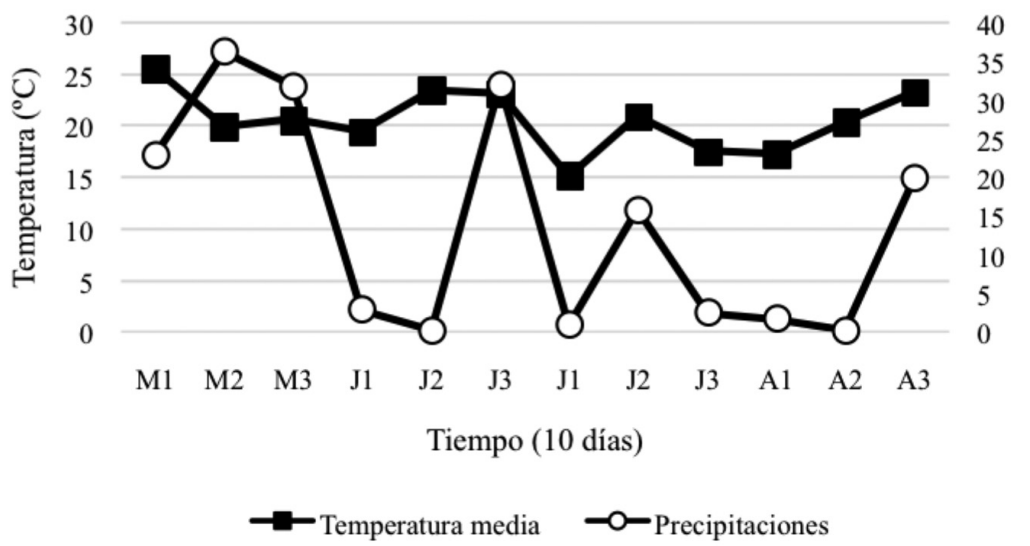

Figura 1. Temperatura media y precipitación en el periodo de mayo a agosto de 2019. 
(m) de largo y de ancho. Como área útil fueron considerados 3,0 m de largo y ancho, descontando $1 \mathrm{~m}$ en cada extremo.

Para la preparación de suelo se adoptaron prácticas culturales convencionales. La siembra se realizó en forma manual con la utilización de una matraca. La emergencia ocurrió 7 días después de la siembra (DDS). Se hizo el raleo a los 15 (DDS) dejándose 8 plantas por metro lineal, 2 en cada hoyo y espaciadas a $0.50 \mathrm{~m}$ entre hileras, con una densidad poblacional aproximada de 160.000 plantas por hectárea. La fertilización mineral se realizó junto con la siembra, en la dosis de $58 \mathrm{~kg} \mathrm{ha}^{-1} \mathrm{P}$ y $60 \mathrm{~kg} \mathrm{ha}^{-1} \mathrm{~K}$, usando como fuente superfosfato triple y cloruro de potasio, respectivamente.

La aplicación de los biofertilizantes se realizó mezclando con la semilla antes de la siembra y posteriormente en cobertura cada 20 días después de la emergencia (DDE), correspondientes a estado de plántula, antes de la floración y en formación de vainas. En total se hicieron 3 aplicaciones, excepto el testigo. La dosis de biofertilizantes que se utilizó fue de $500 \mathrm{ml} / 20$ litros (1) de agua según la recomendación del fabricante.

El control de malezas se hizo en forma manual. Para el control de plagas se utilizó un producto a base de Imidacloprid ( $35 \mathrm{ml} / 201$ de agua) y para el control de enfermedades se usó el fungicida de contacto Oxicloruro de Cobre (30 gramos/20 1 de agua), en forma preventiva, aplicado con pulverizador costal de 201 .

La cosecha se realizó cuando las plantas empezaron a secarse, con el manejo de postcosecha normal del cultivo (trillado, secado y desgrano).

Fueron evaluadas las siguientes variables: Altura de plantas (cm). Se midió en diferentes momentos: a los 30 DDE, 50 DDE y 70 DDE desde la parte superior del suelo hasta la yema apical. Número de vainas por planta (vainaspl-1): se realizó el conteo de las vainas de 5 plantas tomadas al azar dentro del área útil en cada unidad experimental (UE). Número de granos por vaina (granos.vaina ${ }^{-1}$ ): de las vainas cosechadas de 5 plantas fueron contabilizados los granos. Peso de 1000 granos (gr): se contabilizaron mil granos y luego se pesaron para determinar su peso en gramos. Rendimiento de frejol: obtenida por el peso de grano del área útil de cada UE, y posteriormente se extrapolaron en $\mathrm{kg} \mathrm{ha}^{-1}$.

Los datos obtenidos en cada unidad experimental en función de las determinaciones fueron sometidos al análisis de varianza (ANAVA) y los resultados, que fueron significativos, se compararon por el test de Tukey al 5\%.

\section{Resultados y discusión}

Al hacer un análisis de los datos obtenidos en cuanto a la altura de planta a los 30,50 y 70 DDE influenciada por variedades de frejol y biofertilizantes, se observó un efecto significativo según muestran los resultados del test $\mathrm{F}$ (Tabla 1). En la interacción entre los factores no hubo diferencia, excepto a los 70 DDE que revela una diferencia estadística.

La altura de la planta (Tabla 1) para los tres momentos evaluados (30, 50, 70 DDE) arrojó valores superiores en el frejol carioca con relación al frejol negro. Con respecto a los biofertilizantes, se observó que hubo diferencia estadística entre los valores medios de los productos estudiados, y el mayor número de altura se registró con el biofertilizante 2 aplicado. Estos resultados no concuerdan con los expresados por Bermúdez

Tabla 1. Medias de altura de la planta (AP) a los 30, 50 y 70 DDE influenciada por variedades de frejol y biofertilizantes.

\begin{tabular}{|c|c|c|c|}
\hline $\begin{array}{l}\text { Causas de } \\
\text { variación }\end{array}$ & $\begin{array}{c}\text { AP } \\
(30 \mathrm{DDE}) \\
\mathrm{cm}\end{array}$ & $\begin{array}{c}\text { AP } \\
(50 \mathrm{DDE}) \\
\mathrm{cm}\end{array}$ & $\begin{array}{c}\text { AP } \\
(70 \mathrm{DDE}) \\
\mathrm{cm}\end{array}$ \\
\hline \multicolumn{4}{|l|}{ Test F } \\
\hline Variedades (V) & $30,94 *$ & $42,76^{* *}$ & $93,44 * *$ \\
\hline Biofertilizantes (B) & $6,16^{*}$ & $16,57 * *$ & $37,80 * *$ \\
\hline Interacción VxB & $3,00 \mathrm{~ns}$ & $2,48 \mathrm{~ns}$ & $15,00^{* *}$ \\
\hline \multicolumn{4}{|l|}{ Variedades (V) } \\
\hline Frejol carioca & $12,16 \mathrm{a}$ & 26,16 a & 36,33 a \\
\hline Frejol negro & $10,58 \mathrm{~b}$ & $23,75 \mathrm{~b}$ & $33,91 \mathrm{~b}$ \\
\hline \multicolumn{4}{|l|}{ Biofertilizantes (B) } \\
\hline Biofertilizante 2 & $11,75 \mathrm{a}$ & $25,75 \mathrm{a}$ & $36,12 \mathrm{a}$ \\
\hline Biofertilizante 1 & $11,50 \mathrm{ab}$ & $25,00 \mathrm{a}$ & $35,37 \mathrm{~b}$ \\
\hline Testigo & $10,87 \mathrm{~b}$ & $24,12 b$ & $33,87 \mathrm{c}$ \\
\hline C.V. \% (V) & 6,12 & 3,62 & 1,74 \\
\hline C.V. \% (B) & 4,51 & 2,26 & 1,50 \\
\hline Dms 5\% (V) & 0,90 & 1,17 & 0,79 \\
\hline Dms 5\% (B) & 0,68 & 0,75 & 0,70 \\
\hline Media general & 11,37 & 24,95 & 35,12 \\
\hline
\end{tabular}

ns: no significativo; $(*)(* *)$ significativo al 5 y $1 \%$ de probabilidad; Medias seguidas por la misma letra no difieren entre sí por el test de Tukey al 5\%; C.V: Coeficiente de variación; Dms: Diferencia mínima significativa. 
et al. (2011), quienes trabajaron en la efectividad de un biofertilizante foliar sobre el cultivo de frejol común, y determinaron que no existen diferencias en la altura de planta. Estas diferencias podrían explicarse porque los mencionados autores trabajaron con biofertilizantes a base de estiércol de bovino.

La interacción entre variedades de frejol y biofertilizantes en la altura a los 70 DDE fue significativa (Tabla 2). La mayor altura la presenta el frejol carioca. Las variedades se comportan estadísticamente diferentes con los biofertilizantes aplicados, muy por encima del tratamiento sin biofertilizantes. En cuanto a los biofertilizantes, en las variedades se observa un efecto significativo, excepto sin aplicación. El biofertilizante 2 presentó mayor número de altura en el frejol carioca con una media de $37,75 \mathrm{~cm}$ en comparación con el frejol negro. Las aplicaciones de biofertilizantes provocaron incrementos en el crecimiento de la planta en un 11 y $12 \%$, para biofertilizantes 1 y 2, respectivamente. En ese sentido, también algunos estudios mostraron influencia positiva de la aplicación de biofertilizante sobre la altura de plantas en el cultivo de frejol, tomate y maíz (Silva
Tabla 2. Desdoblamiento de la interacción entre las variedades de frejol y biofertilizantes en la altura de la planta a los 70 DDE.

\begin{tabular}{lccc}
\hline & \multicolumn{3}{c}{ Biofertilizantes (b) } \\
\cline { 2 - 4 } Variedades (a) & Testigo & $\begin{array}{c}\text { Biofertilizante } \\
1\end{array}$ & $\begin{array}{c}\text { Biofertilizante } \\
2\end{array}$ \\
\hline Frejol negro & $33,50 \mathrm{Ab}$ & $33,75 \mathrm{~B} \mathrm{ab}$ & $37,50 \mathrm{~B} \mathrm{a}$ \\
Frejol carioca & $34,25 \mathrm{~A} \mathrm{~b}$ & $37,00 \mathrm{~A} \mathrm{a}$ & $37,75 \mathrm{~A} \mathrm{a}$ \\
\hline
\end{tabular}

DMS (Columna): 0,86

DMS (línea): 0,99

Dms: Diferencia mínima significativa. Mismas letras minúsculas en líneas y mayúsculas en columna no difieren entre sí según el test de Tukey al 5\%.

et al., 2011; Medeiros et al., 2011; Sousa et al., 2012). Asimismo, Mamani et al. (2016) señalan que favorece el crecimiento foliar del cultivo de la papa en un $10 \%$ más respecto al testigo ( $\sin$ aplicación).

Como puede observarse en los resultados arrojados por el test de F en la Tabla 3, el número de vainas por planta y el rendimiento fueron

Tabla 3. Medias de número de vainas por planta, granos por vainas, peso de 1000 semillas y rendimiento influenciado por variedades de frejol y biofertilizantes.

\begin{tabular}{|c|c|c|c|c|}
\hline $\begin{array}{l}\text { Causas de } \\
\text { variación }\end{array}$ & $\begin{array}{c}\text { Número de vainas } \\
\text { por planta }\end{array}$ & $\begin{array}{l}\text { Granos } \\
\text { por vainas }\end{array}$ & $\begin{array}{l}\text { Peso de } 1000 \\
\text { semillas (g) }\end{array}$ & $\begin{array}{l}\text { Rendimiento } \\
\left(\mathrm{kg} \mathrm{ha}^{-1}\right)\end{array}$ \\
\hline \multicolumn{5}{|l|}{ Test F } \\
\hline Variedades (V) & $75,00 * *$ & $7,36 \mathrm{~ns}$ & $5376,17 * *$ & $403,33 * *$ \\
\hline Biofertilizantes (B) & $206,14 * *$ & $10,06^{* *}$ & $1,38 \mathrm{Ns}$ & $389,60 * *$ \\
\hline Interacción VxB & $41,57 * *$ & $3,32 \mathrm{~ns}$ & $1,50 \mathrm{Ns}$ & $45,46^{* *}$ \\
\hline \multicolumn{5}{|l|}{ Variedades (V) } \\
\hline Frejol negro & $13,00 \mathrm{~b}$ & $4,48 \mathrm{a}$ & $152,25 \mathrm{~b}$ & 496,66 b \\
\hline Frejol carioca & $13,83 \mathrm{a}$ & $4,33 \mathrm{a}$ & $226,50 \mathrm{a}$ & 551,66 a \\
\hline \multicolumn{5}{|l|}{ Biofertilizantes (B) } \\
\hline Testigo & $11,62 \mathrm{c}$ & $4,25 \mathrm{~b}$ & $188,25 \mathrm{a}$ & $460,50 \mathrm{c}$ \\
\hline Biofertilizante 1 & $14,00 \mathrm{~b}$ & $4,55 \mathrm{a}$ & $189,62 \mathrm{a}$ & 547,12 b \\
\hline Biofertilizante 2 & $14,62 \mathrm{a}$ & $4,42 \mathrm{ab}$ & $190,25 \mathrm{a}$ & $564,87 \mathrm{a}$ \\
\hline C.V. \% (V) & 1,75 & 3,07 & 1,309 & 1,27 \\
\hline C.V. \% (B) & 2,32 & 3,04 & 1,302 & 1,52 \\
\hline DMS (V) & 0,30 & 0,175 & 3,22 & 8,71 \\
\hline DMS (B) & 0,41 & 0,179 & 3,29 & 10,67 \\
\hline Media general & 13,41 & 4,40 & 0,189 & 524,16 \\
\hline
\end{tabular}

ns: no significativo; ** significativo a $1 \%$ de probabilidad; Medias seguidas por la misma letra no difieren entre sí por el test de Tukey al 5\%; C.V: Coeficiente de variación; Dms: Diferencia mínima significativa. 
significativos influenciados por la variedad y el biofertilizante. También se puede apreciar el efecto de las interacciones entre variedades y biofertilizantes.

Al analizar el número de vainas por planta (Tabla 4), se constata que hubo una diferencia de interacción para las variedades y los biofertilizantes estudiados. El mayor número se observa en la variedad de frejol carioca con la aplicación de biofertilizantes, lo cual indica que los tratamientos tienen un mayor efecto sobre la producción de vainas en el cultivo con relación al testigo. En cuanto a los biofertilizantes, el biofertilizante 2 es el que presenta mayor número en la producción de vainas, mientras que el tratamiento (testigo) fue el que registró la menor producción de vainas por planta. Esto evidencia que la utilización de biofertilizantes estimuló una mayor producción de vainas en el cultivo, lo cual indica que los nutrientes que ofrece dicho fertilizante son asimilados exitosamente por la planta. Estos resultados concuerdan con lo expuesto por Escobar et al. (2017), quienes obtuvieron un mayor número de vainas por planta, al aplicar diferentes tipos de biofertilizantes al cultivo de frejol, con efecto promotor y fortalecedor del crecimiento de las plantas, raíces y frutos.

El número de granos por vainas (Tabla 3) fue influenciado por biofertilizantes, que también proporcionaron una diferencia estadísticamente significativa en el cultivo del frejol Según Dourado et al. (2014), el biofertilizante 1 presentó el mayor número de granos con una media de 4,55 , pero estadísticamente no se observa diferencia con el biofertilizante 2 cuyo valor fue de 4,42. Sin embargo difiere del testigo sin la aplicación de biofertilizante

Tabla 4. Desdoblamiento de la interacción entre las variedades de frejol y biofertilizantes en el número de vainas por planta.

\begin{tabular}{lccc}
\hline \multirow{2}{*}{ Variedades } & \multicolumn{3}{c}{ Biofertilizantes } \\
\cline { 2 - 4 } & Testigo & Biofertilizante & Biofertilizante \\
\hline Frejol negro & $12,00 \mathrm{~A} \mathrm{c}$ & $13,00 \mathrm{~B} \mathrm{~b}$ & $14,00 \mathrm{~B} \mathrm{a}$ \\
\hline Frejol carioca & $11,25 \mathrm{~B} \mathrm{~b}$ & $15,00 \mathrm{~A} \mathrm{a}$ & $15,25 \mathrm{~A} \mathrm{a}$ \\
\hline
\end{tabular}

DMS (Columna): 0,43

DMS (línea): 0,58

Dms: Diferencia mínima significativa; (CV) Coeficiente de Variación. Mismas letras minúsculas en líneas y mayúsculas en columna no difieren entre sí según el test de Tukey al 5\%. y es el que mostró la menor cantidad de granos por vainas. Al respecto, Escobar et al. (2017) aseguran que con el uso de compuestos como aminoácidos, fitohormonas y micronutrientes presentes en cada uno de los tratamientos utilizados, se observó significancia estadística con relación al número de granos por vaina en comparación con el testigo absoluto, obteniendo una media general de 5,50 granos.vaina $^{-1}$.

En la variable peso de 1000 semillas (Tabla 3) ocurrieron diferencias significativas para el factor variedad. La mayor cantidad de peso se encuentra en la variedad carioca con una media de 226,50 gramos. En relación con los biofertilizantes, no se observa una diferencia. Estos resultados coinciden con los expresados por Dourado et al. (2014), quienes trabajaron en la acción de bioestimulantes en el desempeño agronómico de maíz y frejol, y concluyeron que no se produjo una diferencia en la variable peso de 1000 semillas.

En cuanto al rendimiento del frejol, tomando en cuenta el factor biofertilizantes (Tabla 5), la respuesta fue diferente en las variedades, y se demostró una mayor eficacia con la aplicación de biofertilizante 2. Este resultado puede ser atribuido a la adecuada disponibilidad de nutrientes minerales que componen el biofertilizante (Cavalcante et al., 2011; Sousa et al., 2012). El suministro apropiado y equilibrado de nutrientes para el frejol puede determinar la elevada productividad (Biscaro et al., 2009).

En relación con las variedades y el uso de biofertilizantes, se constata que el frejol carioca muestra mayor eficiencia en la producción con un

Tabla 5. Desdoblamiento de la interacción entre las variedades de frejol y biofertilizantes en el rendimiento $\left(\mathrm{kg} \mathrm{ha}^{-1}\right)$.

\begin{tabular}{lccc}
\hline \multirow{2}{*}{ Variedades } & \multicolumn{3}{c}{ Biofertilizantes } \\
\cline { 2 - 4 } & Testigo & Biofertilizante & Biofertilizante \\
\hline Frejol negro & $452,50 \mathrm{~B} \mathrm{c}$ & $501,00 \mathrm{~B} \mathrm{~b}$ & $536,50 \mathrm{~B} \mathrm{a}$ \\
\hline Frejol carioca & $468,50 \mathrm{~A} \mathrm{~b}$ & $593,25 \mathrm{~A} \mathrm{a}$ & $593,25 \mathrm{~A} \mathrm{a}$ \\
\hline
\end{tabular}

DMS (Columna): 11,51

DMS (línea): 15,09

Dms: Diferencia mínima significativa; (CV) Coeficiente de Variación. Mismas letras minúsculas en líneas y mayúsculas en columna no difieren entre sí según el test de Tukey al 5\%. 
valor de 593,25 $\mathrm{kg} \mathrm{ha}^{-1}$. Los altos rendimientos obtenidos en los tratamientos con biofertilizante se pueden explicar por la utilización de componente orgánico, lo cual hace que la planta disponga de todos los nutrientes en las raíces (Gómez et al., 2008).

Al realizar una comparación entre el testigo (sin aplicación) y la aplicación de biofertilizantes 1 y 2 , se comprueba que en la producción de frejol carioca hubo una diferencia de $21 \%$. Estos resultados coinciden con los obtenidos por Silva et al. (2013), quienes observaron un efecto significativo de la aplicación de biofertilizante, con un aumento de $21 \%$ en comparación con el testigo. Beltrão Júnior et al. (2012) verificaron un incremento en la productividad de frejol al utilizar biofertilizante con fuente de fertilizante orgánica. Los biofertilizantes proporcionan mejoras en las propiedades físicas, químicas y biológicas del suelo y cuando son aplicados sobre las hojas pueden contribuir al suministro equilibrado de macro y micronutrientes (Rodrigues et al., 2009; Patil, 2010). Esto permite que el cultivo desarrolle todo su potencial productivo (Oliveira et al., 2014).

\section{Conclusiones}

Este experimento demuestra la eficacia de los biofertilizantes en la producción de variedades de frejol, por cuanto su aplicación influyó de forma significativa en la altura de planta, número de vainas por planta, granos por vainas, peso de 1000 semillas y rendimiento.

Se observaron interacciones significativas de los factores estudiados, en la altura de planta a los $70 \mathrm{DDE}$, número de vainas por planta y rendimiento. La mejor combinación se obtuvo con el frejol carioca y el biofertilizante 2 .

\section{Literatura Citada}

Andrade, C.A.B.; Patroni, S.M.S.; Clemente, E.; Scapim, C.A. 2004. Produtividade e qualidade nutricional de cultivares de feijão em diferentes adubações. Ciência e Agrotecnologia, 28(5): 1077-1086

Beltrão Junior, J.A.; Cruz; J.S.; Coelho de Sousa, E.C.; Da

Silva, L.A.S.

2012. Rendimento do feijão-caupi adubado com diferentes doses de biofertilizante orgânico produziodo a través da biodegadação acelerada de residuos do coqueiro no município de Trairí - CE. Irriga, Edição Especial: 423-437.

Bermúdez, Y.M; Álvarez, A.M; Luna, B.G.

2011. Efectividad de un biofertilizante foliar sobre el cultivo de frijol común (Phaseolus vulgaris), Ciencia e Interculturalidad, 8(1): 128-140.

Biscaro, G.A.; Goulart Júnior, S.A.R.; Soratto, R.P.; Freitas

Júnior, N.A.; Motomiya, A.V.A.; Calado Filho, G.C.

2009. Molibdênio via semente e nitrogênio em cobertura no feijoeiro irrigado em solo de cerrado. Ciência e Agrotecnologia, Lavras, 33(5): 1280-1287.

Carvajal, M.J.S.; Mera, B.A.C.

2010. Biological fertilization: state of the art techniques for a sustainable agricultural development. Producción + Limpia, (5):2. 77-96

Cavalcante, L.F.; Vieira, M.S.; Santos, A.F.; Oliveira, W.M.; Nascimento, J.A.M.

2010. Água salina e esterco bovino líquido na formação de mudas de goiabeira cultivar Paluma. Revista Brasileira de Fruticultura, 32: 251-261.

$\mathrm{DMH}$

2018. Datos de los parámetros meteorológicos, Paraguay. (Dirección de Meteorología e Hidrología). https://www. meteorologia.gov.py/emas/.

Dourado, N.D.; Dario, G.J.A.; Barbieri, A.P.P.; Martin, T.N. 2014 Ação de bioestimulante no desempenho agronômico de milho e feijão. Bioscience Journal, 30: 371-379.
Escobar, O.W.S.; Recalde, V.T.; Jaime A. Pazmiño, M.J.A.; Vivas, V.R.

2017. Respuesta del cultivo de frejol caraota (Phaseolus vulgaris L.) a la aplicación foliar complementaria de tres bioestimulantes. Dominio de las Ciencias, 3(3): 556-571.

Galbiatti, J.A.; Da Silva, F.G.; Franco, C.F.

2011. Desenvolvimento do feijoeiro sob o uso de biofertilizante e adubação mineral. Eng. Agríc., 31(1): 167-177.

Gómez, M.N.O.; Sierra, M.M.; González, C.M.; Cantu, A.M.A.; Ramírez, F.A.; Wong, P.J.; Manjarrez, S.M.; \& Ramírez, D.J.L.; Espinosa, C.A.

2008. Híbrido de Maíz de alto rendimiento para el trópico húmedo y seco de México. Agricultura técnica, 34(1): 101-105.

León, S.I.; Faure, A.B.; Rodríguez, M.O.; Benítez, G.R.; Suárez, G.Y.; Rodríguez, R.R.

2008. Selección de nuevas variedades de frijol común (Phaseolus vulgaris $\mathrm{L}$.) frente a las principales enfermedades del cultivo en Cuba. FITOSANIDAD, 12(1): 27-31.

López, O.E.; González, E.; de Llamas, P.A.; Molinas, A.S.;

Franco, E.; D; E.S.; García, S.; Ríos, E.

1995. Reconocimiento de suelos y capacidad de uso de las tierras; Región Oriental. Paraguay. MAG/Dirección de Ordenamiento Ambiental. Proyecto de comercialización de Uso de la Tierra. Convenio 3445. P. Banco Mundial. 28 p.

Mamani, E.; Morales, V.; Ortuño, N.

2016. Aplicación de biofertilizantes foliares en el cultivar Huaycha (Solanum tuberosum subsp. andigena) en los valles interandinos de Bolivia. Revista Latinoamericana de la Papa, 20(2): 14-25.

Medeiros, M.B.; Wanderley, P.A.; Franklin, F.; Fernandes, F.S.; Alves, G.R.; Dantas, P.; Cordão, R.P.; Xavier, W.M.R.; Leal Neto, J.S.

2003. Uso de biofertilizantes líquidos no manejo ecológico de pragas agrícolas. In: Encontro Temático Meio Ambiente 
e Educação Ambiental da UFPB, 2., 2003, João Pessoa. Anais... João Pessoa, 2003. Pp. 19-23.

Medeiros, R.F.; Cavalcante, L.F.; Mesquita, F.O.; Rodrigues, R.M.; Sousa, G.G.; Diniz, A.A.

2011. Crescimento inicial do tomateiro-cereja sob irrigação com águas salinas em solo com biofertilizantes bovino. Revista Brasileira de Engenharia Agrícola e Ambiental, 15(5): 505-511.

Melém Júnior, N.J.; Brito, O.R.; Fonsceca Júnior, N.S.; Fonsceca, I.C.B.; Aguiar, S.X.

2011. Nutrição mineral e produção de feijão em áreas manejadas com e sem queima de resíduos orgânicos e diferentes tipos de adubação. Semina: Ciências Agrárias, 32(1): 7-18.

Oliveira, J.R.; Gomes, L.R.F.; Araújo, A.S.F.; Martini, S.F.; Lopes, J.B.; Araújo, R.M.

2014. Estado nutricional e produção da pimenteira com uso de biofertilizantes líquidos. Revista Brasileira de Engenharia Agrícola e Ambiental, 18(12): 1241-1246.

Patil, N.M.

2010 Biofertilizer effect on growth, protein and carbohydrate content in stevia rebaudiana var bertoni. Recent Research in Science and Technology, 2: 42-44.
Rodrigues, A.C.; Cavalcante, L.F.; Oliveira, A.P. de; Sousa, J.T. de; Mesquita, F.O.

2009. Produção e nutrição mineral do maracujazeiro-amarelo em solo com biofertilizante supermagro e potássio. Revista Brasileira de Engenharia Agrícola e Ambiental, 13: 117-124.

Silva, F.L.B.; Lacerda, C.F.; Never, A.L.R.; Sousa, G.G.; Sousa, C.H.C.; Ferreira, F.J.

2013. Irrigação com águas salinas e uso de biofertilizante bovino nas trocas gasosas e produtividade de feijão-decorda. Irriga, 18(2): 304- 317.

Silva, F.L.B.; Lacerda, C.F.; Sousa, G.G.; Neves, A.L.R.; Silva, G.L.; Sousa, C.H.C.

2011. Interação entre salinidade e biofertilizante bovino na cultura do feijão-de-corda. Revista Brasileira de Engenharia Agrícola e Ambiental, 15(4): 383-389.

Sousa, G.G.; Marinho, A.B.; Albuqueruqe, A.H.P.; Viana, T.V.A.; Azevedo, B.M.

2012. Crescimento inicial do milho sob diferentes concentrações de biofertilizante bovino irrigado com águas salinas. Revista Ciência Agronômica, Fortaleza, 43(2): 237-245. 
